\section{Utilidad pronóstica del PET/CT en cáncer de páncreas}

\author{
DAVID LADRÓN DE GUEVARA ${ }^{1}$, GONZALO PAVEZ ${ }^{2, a}$, \\ JAIME ZAPATA ${ }^{3, b}$, CLAUDIO ROMERO ${ }^{3, b}$, VALEZKA TAPIA ${ }^{3, b}$, \\ ERWIN BUCKEL ${ }^{4}$, MARIO FERRARIO ${ }^{4}$
}

\section{Prognostic value of PET/CT in pancreatic cancer}

Background: Pancreatic cancer is the tenth most prevalent cancer in world, and represents the fourth cause of cancer death. It has a five year-survival of 5\%. Aim: To assess the prognostic value of PET/CT in pancreatic cancer. Material and Methods: Sixty-nine patients with pancreatic adenocarcinoma who underwent staging ${ }^{18}$ F-fluorodeoxyglucose (FDG) PET/CT between December 2008 and July 2016 were selected. Gender, age, body-mass index, laboratory tests (Ca 19-9, hemoglobin, erythrocyte sedimentation rate, liver enzymes, lactate dehydrogenase), histological differentiation of tumor, American Joint Committee on Cancer (AJCC) stage, size and ${ }^{18} F-F D G$ uptake (maximal standardized uptake value [SUVmax]) of the primary tumor, nodal involvement and distant metastasis detected by PET/CT were registered. Survival was assessed using Kaplan-Meier curves, Log Rank test and Cox multivariable analysis. Results: Mortality was 66.7\%, during a mean observation time of 18 months (range 20 days-66 months). Curative surgery, lack of metastases detected by PET/CT, histologically well differentiated tumors, and SUVmax $\leq 4.3$ were significantly associated with a better specific survival, determined by the Log Rank test. Histological differentiation was the only variable that had a statistically significant prognostic value in the multivariable analysis. Conclusions: The detection of distant metastases and the intensity of primary tumor ${ }^{18} \mathrm{~F}-\mathrm{FDG}$ uptake during PET/CT provide useful prognostic information in pancreatic cancer patients.

(Rev Med Chile 2018; 146: 413-421)

Key words: Carcinoma, Pancreatic Ductal; Computed Tomography; Medical Oncology; Pancreatic Neoplasm; Positron Emission Tomography.

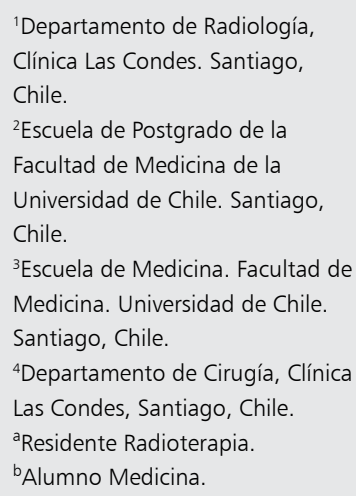

Los autores declaran no tener conflictos de interés.

Trabajo no recibió

financiamiento.

Recibido el 24 de septiembre de 2017, aceptado el 20 de febrero de 2018.

Correspondencia a Dr. David Ladrón de Guevara H. Departamento de Radiología, Clínica Las Condes. Santiago, Chile. Fono: 56222105174 dlg@clc.cl
E 1 cáncer de páncreas es la décima neoplasia maligna en prevalencia, y representa la cuarta causa de mortalidad por cáncer en Estados Unidos de Norteamérica, con un riesgo de desarrollarlo de $1,5 \%$ durante el período vital ${ }^{1}$. Cerca de 330.000 personas fallecieron por cáncer de páncreas en el año 2012 en el mundo ${ }^{2}$. En Chile, según datos de GLOBOCAN, se espera que para 2020, 1.524 pacientes sean diagnosticadas con esta enfermedad ${ }^{2}$.

La sobrevida en cáncer de páncreas llega a ser dramáticamente baja, hasta $5 \%$ a 5 años, lo que se atribuye a su alta letalidad y presentación tardía ${ }^{3}$. Al tiempo del diagnóstico, solo $20 \%$ se llegan a tratar con intención curativa ${ }^{4}$. En aquellos tumores de cabeza pancreática que han sido diagnosticados precozmente y operados, la sobrevida global a 5 años llega a ser de hasta $27 \%{ }^{5}$. No obstante, para casos avanzados, la sobrevida no sobrepasa los 9-15 meses, y en casos de enfermedad metastásica, los 3 y 6 meses $^{6}$.

El principal factor pronóstico en cáncer de páncreas es el grado de avance de la enfermedad, tanto de la invasión local como de metástasis a 
distancia ${ }^{7}$. Es por esto que las imágenes, principalmente tomografía computada (TC) y resonancia magnética y la exploración quirúrgica son las herramientas principales en la valoración pronóstica.

La tomografía por emisión de positrones-tomografía computada (PET/CT) utilizando flúor18-fluorodeoxiglucosa (F18-FDG) es una técnica híbrida muy utilizada en oncología, tanto en etapificación, como en evaluación de tratamiento y seguimiento. Sin embargo, no forma parte del estudio imagenológico convencional en la etapificación de cáncer de páncreas, y por lo tanto hay pocos datos disponibles sobre su utilidad pronóstica. A pesar de esto, la escasa literatura disponible sugiere que el PET/CT aportaría información pronóstica, particularmente en relación al grado de captación de F18-FDG que presenta la lesión primaria $^{8-10}$. Esto se debería a que los tumores biológicamente más agresivos suelen presentar mayor avidez de F18-FDG, hecho que determina su utilidad como marcador biológico en diversos tipos de neoplasias ${ }^{11}$. Para medir esta captación, se han utilizado índices semicuantitativos que traducen la intensidad de captación de FDG en el tumor primario (valor de captación estándar: SUV), la masa tumoral activa (volumen tumoral metabólico: VTM), o una combinación de ambos (masa tumoral glicolítica: MTG) ${ }^{8,12,13,14}$.

En un reciente metaanálisis sobre la utilidad pronóstica del PET/CT en cáncer pancreático, Zhu et $\mathrm{al}^{15}$ recopilan la experiencia de 16 estudios en un total de 1.146 pacientes, $y$ a pesar de la heterogeneidad de los resultados encontrados, concluyen que el valor de captación estándar máximo (SUVmax), VTM y MTG pudieran ser factores pronósticos significativos en estos pacientes. En nuestra revisión bibliográfica, no encontramos publicaciones referentes a la experiencia local ni regional en la evaluación de la utilidad pronóstica del PET/CT en cáncer pancreático.

El objetivo de este estudio es estimar la utilidad pronóstico de sobrevida del PET/CT con F18-FDG en pacientes con cáncer de páncreas recientemente diagnosticado.

\section{Pacientes y Método}

Se trata de un estudio pronóstico retrospectivo. Para la selección de pacientes se consultó la base de datos de PET/CT de Clínica Las Condes.

Se seleccionaron los pacientes derivados por tumor o cáncer de páncreas en etapificación entre diciembre de 2008 y julio de 2016, que tuvieran confirmación histopatológica de adenocarcinoma de páncreas. Se excluyeron los tumores con histología distinta al adenocarcinoma (linfoma, tumor de células de los islotes, etc). Los pacientes extranjeros y no residentes en Chile fueron excluidos debido a la imposibilidad de averiguar su estado vital.

Se revisaron las imágenes del PET/CT registrándose: SUVmax del tumor primario; número y ubicación de ganglios linfáticos comprometidos; $\mathrm{y}$ ubicación de las metástasis a distancia. Se consideró metástasis a distancia a la siembra secundaria no ganglionar, tal como hepática, peritoneo-omental, ósea, pulmonar, pleural, u otra localización lejana al tumor.

Se revisó la ficha clínica institucional rescatando la siguiente información: peso, talla, índice de masa corporal (IMC), etapificación clínica y patológica según American Joint Commettee on Cancer (AJCC) ${ }^{16}$, exámenes de laboratorio (Ca 199, hemoglobinemia, velocidad de eritrosedimentación [VHS], transaminasa glutámico oxaloacética [TGO], trasnaminasa glutámico pirúvica [TGP], fosfatasas alcalinas [FFAA], deshidrogenasa láctica $[\mathrm{LDH}])$, lipasa), grado de diferenciación a la histopatología. También se registró el tipo de tratamiento efectuado, ya sea cirugía curativa, quimioterapia, radioterapia, o ambos.

Para evaluar la utilidad pronóstica de la clasificación AJCC se separaron a los pacientes en etapas 0, I , II, III y IV, y en subetapas 0, IA, IB, IIA, IIB, III y IV.

El estado vital se indagó mediante solicitud de certificados de defunción al Servicio de Registro Civil e Identificación de Chile. Se calificaron como fallecidos para efectos de determinación de supervivencia cáncer-específica, aquellos pacientes que presentaron "cáncer de páncreas" como causa de muerte primaria o secundaria. Aquellos pacientes con fallecimiento de otra causa fueron considerados vivos hasta ese momento. Dichos pacientes y aquellos no fallecidos durante el tiempo de observación fueron considerados como "censurados" para los efectos del análisis de sobrevida.

\section{Protocolo de FDG-PET/CT y medición de parámetros}

El examen PET/CT se realizó en equipo GE Discovery STE 16 canales (GE Healthcare, Wakes- 
ha, WI, EE. UU.) en todos los casos, 1 h después de administrar F18-FDG en dosis de $0,1 \mathrm{mCi} / \mathrm{Kg}$ por vía endovenosa. Los pacientes fueron citados en ayunas (al menos $6 \mathrm{~h}$ ). El examen se realizó solo en pacientes con glicemias menores a $200 \mathrm{mg} /$ dl. En caso de glicemias mayores a $200 \mathrm{mg} / \mathrm{dl} \mathrm{se}$ administró suero fisiológico $500-1.000 \mathrm{ml}$, hasta alcanzar glicemias adecuadas.

Inmediatamente después del PET/CT, y sin mover al paciente de la camilla del PET/CT, se realizó TC de cerebro, cuello, tórax, abdomen y pelvis en todos los casos, previa administración de medio de contraste iodado endovenoso. El examen fue interpretado por un especialista doble radiólogo/ médico nuclear.

Para efectos de este estudio se registró tamaño (mm), VTM (cc) y SUVmax y SUVpromedio del tumor pancreático, número y ubicación de adenopatías, y localización de metástasis a distancia. Para la estimación de la captación se utilizará el índice SUVmax, que equivale a la actividad de una lesión dividida por el volumen conocido de un vóxel según la fórmula:

SUVmax: $\frac{\text { Actividad en una lesión }(\mathrm{Bq}) / \text { Volumen píxel }(\mathrm{ml})}{\text { Actividad inyectada }(\mathrm{Bq}) / \text { Peso pcte }(\mathrm{g})}$
El VTM se obtuvo dibujando un área de interés $3 \mathrm{D}$ alrededor del tumor pancreático, con selección automática de los vóxel con $\mathrm{SUV} \max \geq 2,5$ según método de Lee et $\mathrm{al}^{13}$ (Figura 1). La MTG se estimó de acuerdo a la fórmula VTM x SUVpromedio según lo publicado previamente ${ }^{13}$.

\section{Análisis estadístico}

El diseño muestral es no probabilístico consecutivo, que cumpla con los criterios de inclusión.

Para comparación de variables cuantitativas entre grupos fallecidos vs no fallecidos se utilizaron pruebas paramétricas (t-test) y no paramétricas (Mann-Whitney), según correspondiera. Para estudiar variables cualitativas se utilizó el test exacto de Fischer. La sobrevida específica se estudió utilizando curvas de Kaplan-Meier, log-rank test, y regresión multivariable de Cox, pruebas que se aplicaron a cada una de las variables clínicas, de laboratorio, histopatológicas y del PET/CT.

\section{Aspectos éticos}

La base de datos de PET/CT de la cual fueron seleccionados los casos cuenta con la aprobación para su utilización con estos fines por el Comité

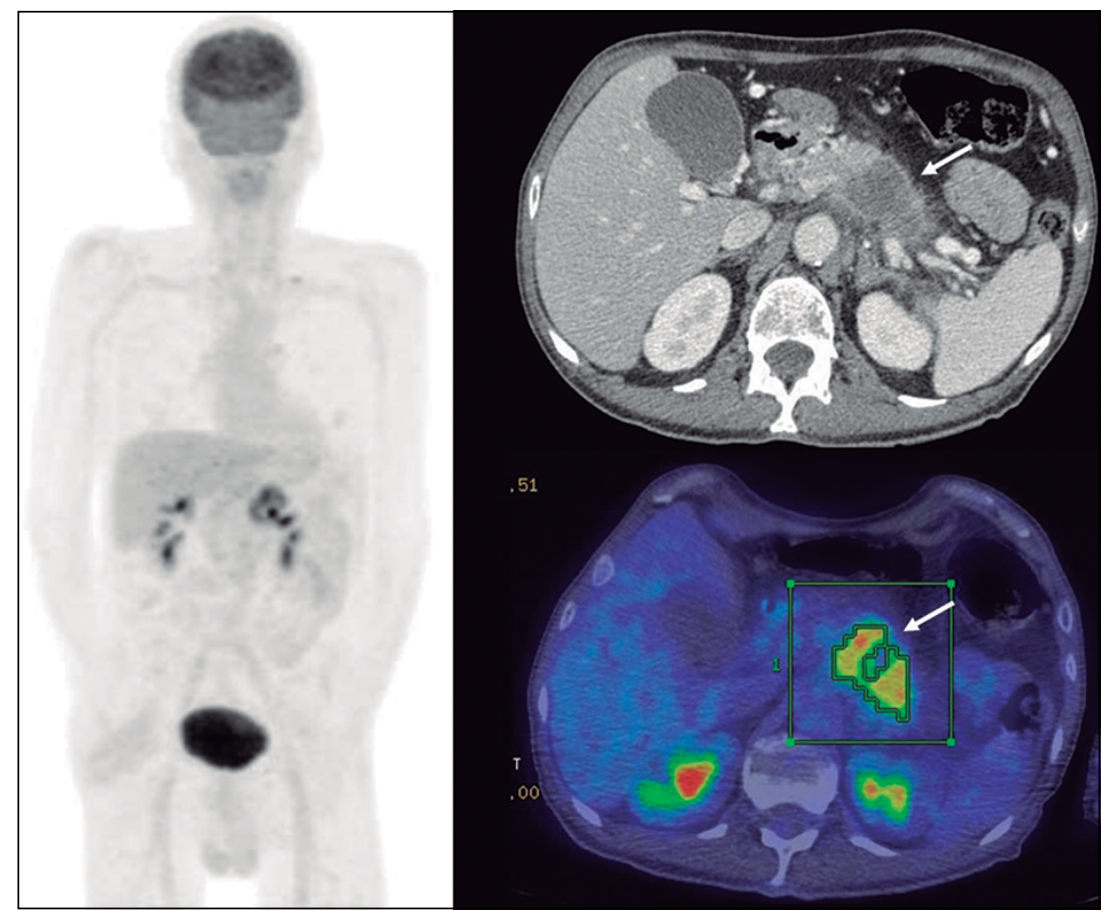

Figura 1. Paciente en etapificación por cáncer de páncreas. Imagen superior derecha: tomografía computada contrastada que muestra extensa lesión hipodensa en cuerpo y cola pancreática (flecha), que infiltra vasos esplénicos. Imagen inferior derecha: PET/CT muestra lesión pancreática parcialmente necrótica con VTM de 30,4 cc y SUVmax: 7,4. Imagen izquierda: Maximun intensity projection (MIP) del PET con lesión hipercaptante pancreática, sin metástasis evidentes. 
de Ética de la institución. Todos los pacientes incluidos en este estudio han adherido y firmado un consentimiento informado para realizar investigación utilizando los estudios de PET/CT. Para efectos del análisis de los datos y la publicación del estudio, se usó la información codificada. Se protegió la privacidad y confidencialidad de la información de los pacientes del estudio.

\section{Resultados}

De un total de 69 pacientes con cáncer de páncreas, $66,7 \%$ falleció por causa del cáncer pancreático durante el seguimiento, con un tiempo de observación promedio de 18 meses (rango 20 días-66 meses). Dos pacientes fallecieron por causa no relacionada directamente al cáncer pancreático, los que fueron observados como vivos hasta ese momento.

La comorbilidad, laboratorio, características del tumor pancreático y tratamientos efectuados a los pacientes se muestra en la Tabla 1 . Al comparar entre grupos de pacientes según estado vital, se encontró que los pacientes fallecidos presentaron niveles significativamente mayores de LDH (p: 0,024) y Ca19-9 (p: 0,045), niveles menores de lipasa (p: 0,039), mayor frecuencia de metástasis a distancia en el PET/CT (p: 0,038), menor frecuencia de cirugías curativas ( $\mathrm{p}: 0,027$ ) y mayor frecuencia de uso de quimioterapia (p: 0,002) (Tabla 1).

Al utilizar curvas de Kaplan-Meier y log-rank test se demostró mayor sobrevida en pacientes operados con criterio curativo (mediana 67,8 meses, 24,2-111,2 m, IC 95\%) vs aquellos tratados en forma paliativa (mediana 11,2 meses, 7,4-15,0 m, IC 95\%) (p: 0,001), en pacientes sin metástasis a distancia al PET/CT (mediana 24,8 meses, 7,2-42,3 m, IC 95\%) vs metastásicos (mediana 12,5 meses, 9,3-15,6 m, IC 95\%) (p: 0,015), en tumores bien diferenciados vs moderada vs mal diferenciados (log-rank test de tendencia: Chi cuadrado: 7,019, p: 0,03), y en tumores con SUVmax $\leq 4,3$ (mediana 22,9 meses, 7,2-37,0 m, IC95\%) vs tumores de mayor captación (mediana 12,8 meses, 8,0-17,5 m, IC 95\%) (p: 0,045). Las curvas de Kaplan-Meier se muestran en Figuras 2, 3, 4 y 5 , respectivamente.

El análisis de regresión multivariable solo mostró significación para el grado de diferencia- ción histológico del adenocarcinoma de páncreas ( $p: 0,025$ ), con mayor sobrevida en tumores bien/ moderado vs mal diferenciados.

\section{Discusión}

La posibilidad de establecer una estimación pronóstica de una enfermedad determinada es de suma importancia para la toma de decisiones en salud. Constituye información relevante para el paciente y sus familiares, y es indispensable al momento de plantear opciones terapéuticas y planificar adecuadamente la administración de recursos ${ }^{17}$.

El principal factor pronóstico a considerar en adenocarcinoma de páncreas es el grado de avance de la enfermedad al momento del diagnóstico, lo que condiciona a su vez la opción de tratamiento curativo, cual es la cirugía ${ }^{7}$. La diseminación a distancia determina generalmente irresecabilidad, sin posibilidad de curación. Esto se ve reflejado en nuestros resultados, donde las variables cirugía curativa y ausencia de metástasis visibles al PET/ CT se asocian a una mejor sobrevida específica utilizando curvas de Kaplan-Meier. La falta de significación pronóstica del estadio AJCC puede ser explicada en parte por la división en etapas (0, I, II, III y IV) y subetapas, lo que podría restar significación en nuestro grupo relativamente pequeño de pacientes.

El grado de diferenciación tumoral del adenocarcinoma a la histopatología, y la intensidad de captación de F18-FDG medido mediante SUVmax también presentaron valor pronóstico de sobrevida, lo que es concordante con la literatura ${ }^{8-10}$. Estas dos variables han mostrado alta correlación en una variedad de tumores malignos, por cuanto a mayor desdiferenciación tumoral, mayor índice proliferativo y agresividad del cáncer, lo que se asocia a mayor expresión de receptores GLUT y consumo de glucosa ${ }^{11,18}$. Ya que la intensidad de captación de F18-FDG es considerado un marcador biológico de actividad y agresividad del tumor ${ }^{11}$, y en el caso del cáncer de páncreas parece tener valor pronóstico, creemos que pudiera ser considerada al momento de plantear las opciones terapéuticas y sobrevida estimada, especialmente en el grupo de pacientes sin metástasis al momento del diagnóstico. Esto porque quizás aquellos tumores de alto grado histológico o con captaciones altas 
Utilidad pronóstica del PET/CT en cáncer de páncreas - D. Ladrón de Guevara et al

Tabla 1. Comorbilidad, laboratorio y características del tumor según estado vital

\begin{tabular}{|c|c|c|c|}
\hline & Fallecidos $(n=46)$ & No fallecidos $(n=23)$ & $\mathbf{p}$ \\
\hline Edad (años) & $66,6 \pm 10,3$ & $62,5 \pm 12,4$ & NS* \\
\hline Hombres & $28(60,9 \%)$ & $13(56,5 \%)$ & $N S^{* *}$ \\
\hline IMC & $24,6 \pm 4,6$ & $25,6 \pm 4,1$ & NS* \\
\hline Diabetes mellitus & $21(45,7 \%)$ & $7(30,4 \%)$ & $N S^{* *}$ \\
\hline Tabaco & $6(13,0 \%)$ & $8(34,8 \%)$ & $N S^{* *}$ \\
\hline \multicolumn{4}{|l|}{ Laboratorio } \\
\hline Glicemia [mg/100 mL] & $121 \pm 36,4$ & $103,71 \pm 16,42$ & NS* \\
\hline Hemoglobina [Mg/dL] & $12,96 \pm 2,1$ & $13,87 \pm 1,91$ & NS* \\
\hline VHS $[\mathrm{mm} / \mathrm{h}]$ & $31[1-140]$ & $28[2-74]$ & $N S^{* * *}$ \\
\hline Bilirrubina [mg/dL] & $1,0[0,1-21,1]$ & $0,7[0,2-7,4]$ & $N S^{* * *}$ \\
\hline Amilasa [mU/mL] & $43[5-503]$ & $113[50-505]$ & $N S^{* * *}$ \\
\hline FFAA $[\mathrm{mU} / \mathrm{mL}]$ & $122[1-933]$ & $91[39-870]$ & $N S^{* * *}$ \\
\hline $\mathrm{GOT}[\mathrm{mU} / \mathrm{mL}]$ & $43[17-347]$ & $53[19-181]$ & $N S^{* * *}$ \\
\hline $\mathrm{GPT}[\mathrm{mU} / \mathrm{mL}]$ & $48,5[16-641]$ & $47,5[16-260]$ & $N S^{* * *}$ \\
\hline Lipasa [U/mL] & $29,0[14-656]$ & $91,5[29-1.558]$ & p: $0,039^{* * *}$ \\
\hline LDH [Ui/L] & 223 [126-3.132] & $181[10-308]$ & p: $0,024^{* * *}$ \\
\hline CA 19-9 [U/mL] & $376[2-140.000]$ & $82[2-30.778]$ & p: $0,045^{* * *}$ \\
\hline \multicolumn{4}{|l|}{$\mathrm{PET} / \mathrm{CT}$} \\
\hline Tamaño (mm) & $40,6 \pm 18,7$ & $40,0 \pm 25,6$ & NS* \\
\hline SUVmax & $8,5 \pm 4,3$ & $8,1 \pm 5,0$ & NS* \\
\hline VTM & $21,9 \pm 23,5$ & $23,0 \pm 31,8$ & $N S^{* * *}$ \\
\hline MTG & $226,2 \pm 294,9$ & $300,5 \pm 538,2$ & $N S^{* * *}$ \\
\hline Metástasis ganglionares & $30(65,2 \%)$ & $11(47,8 \%)$ & $N S^{* *}$ \\
\hline Metástasis a distancia & $31(67,4 \%)$ & $9(39,1 \%)$ & p: $0,0380^{* *}$ \\
\hline Hepáticas & $22(47,8 \%)$ & $7(30,4 \%)$ & $N S^{* *}$ \\
\hline Peritoneales & $19(41,3 \%)$ & $5(21,7 \%)$ & $N S^{* *}$ \\
\hline Pulmonares & $10(21,7 \%)$ & $3(13,0 \%)$ & $N S^{* *}$ \\
\hline Óseas & $4(8,7 \%)$ & $0(0 \%)$ & $N S^{* *}$ \\
\hline Otras & $3(6,5 \%)$ & $0(0 \%)$ & $N S^{* *}$ \\
\hline \multicolumn{4}{|l|}{ Histopatología } \\
\hline Bien diferenciado & $1(2,2 \%)$ & $3(13,0 \%)$ & $N S^{* *}$ \\
\hline Moderadamente diferenciado & $15(32,6 \%)$ & $7(30,4 \%)$ & $N S^{* *}$ \\
\hline Mal diferenciado & $7(15,2 \%)$ & $5(21,7 \%)$ & $\mathrm{NS}^{* *} \mathrm{NS}^{\dagger}$ \\
\hline No consignado & $23(50,0 \%)$ & $8(34,8 \%)$ & $N S^{* *}$ \\
\hline \multicolumn{4}{|l|}{ Etapa AJCC } \\
\hline 1 & $3(6,5 \%)$ & $5(21,7 \%)$ & $N S^{* *}$ \\
\hline 2 & $7(15,2 \%)$ & $5(21,7 \%)$ & $N S^{* *}$ \\
\hline 3 & $3(6,5 \%)$ & $1(4,3 \%)$ & $N S^{* *}$ \\
\hline 4 & $33(71,7 \%)$ & $12(52,2 \%)$ & $\mathrm{NS}^{* *} \mathrm{NS}^{\dagger}$ \\
\hline \multicolumn{4}{|l|}{ Tratamiento } \\
\hline Cirugía curativa & $6(13,0 \%)$ & $9(39,1 \%)$ & $0,027 * *$ \\
\hline Quimioterapia & $37(80,4 \%)$ & $10(43,5 \%)$ & $0,002^{* *}$ \\
\hline Radioterapia & $7(15,2 \%)$ & $3(13,0 \%)$ & $N S^{* *}$ \\
\hline
\end{tabular}

IMC: Índice de masa corporal; VTM: Volumen tumoral metabólico; MTG: Masa tumoral glicolítica. Test estadísticos: *T-test; **Test exacto de Fischer; ${ }^{* * *}$ Test de Mann-Whitney. ${ }^{\dagger}$ Chi cuadrado en análisis de más de dos grupos. 

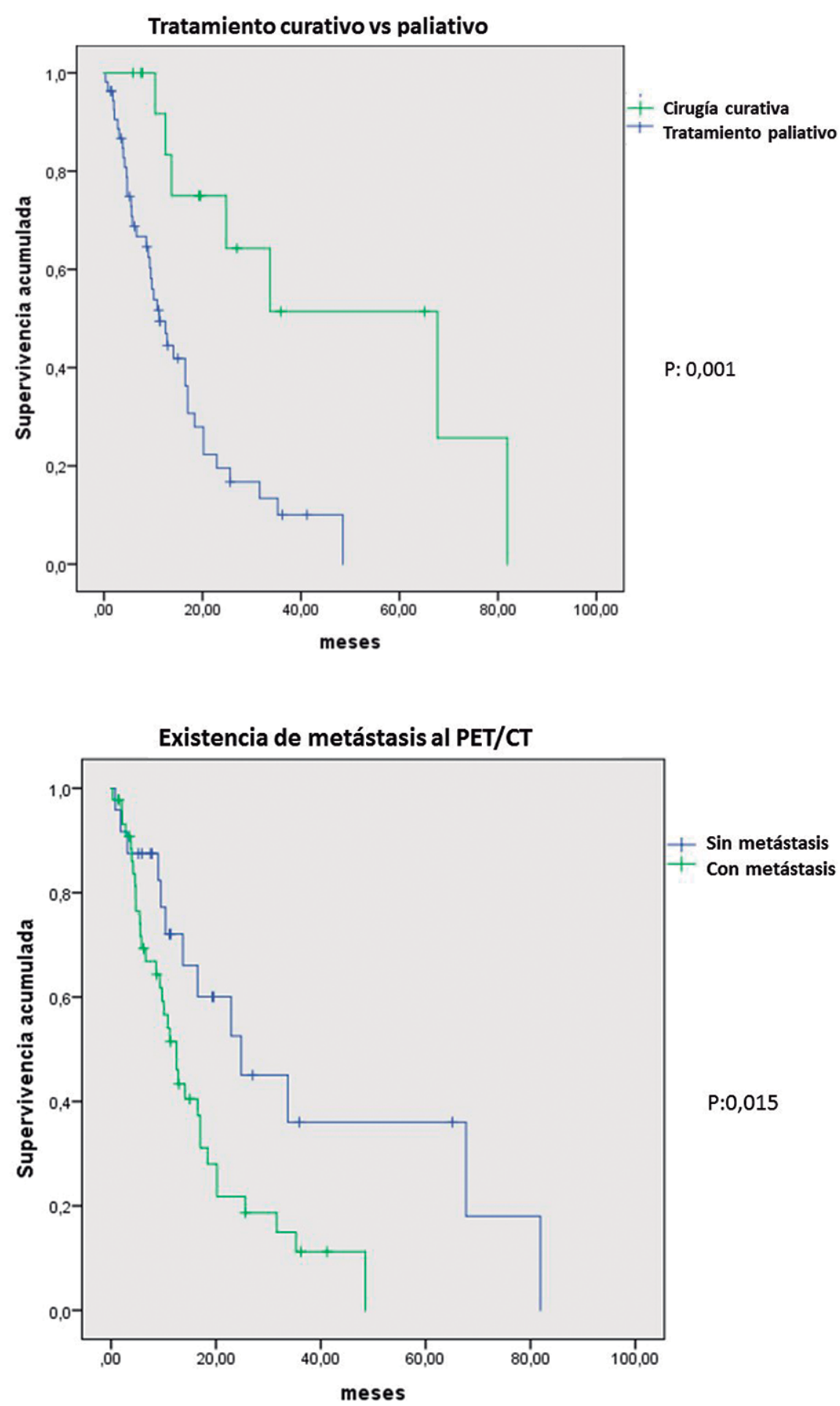

Figuras 2 y 3. Curvas de Kaplan-Meier según factor pronóstico. 

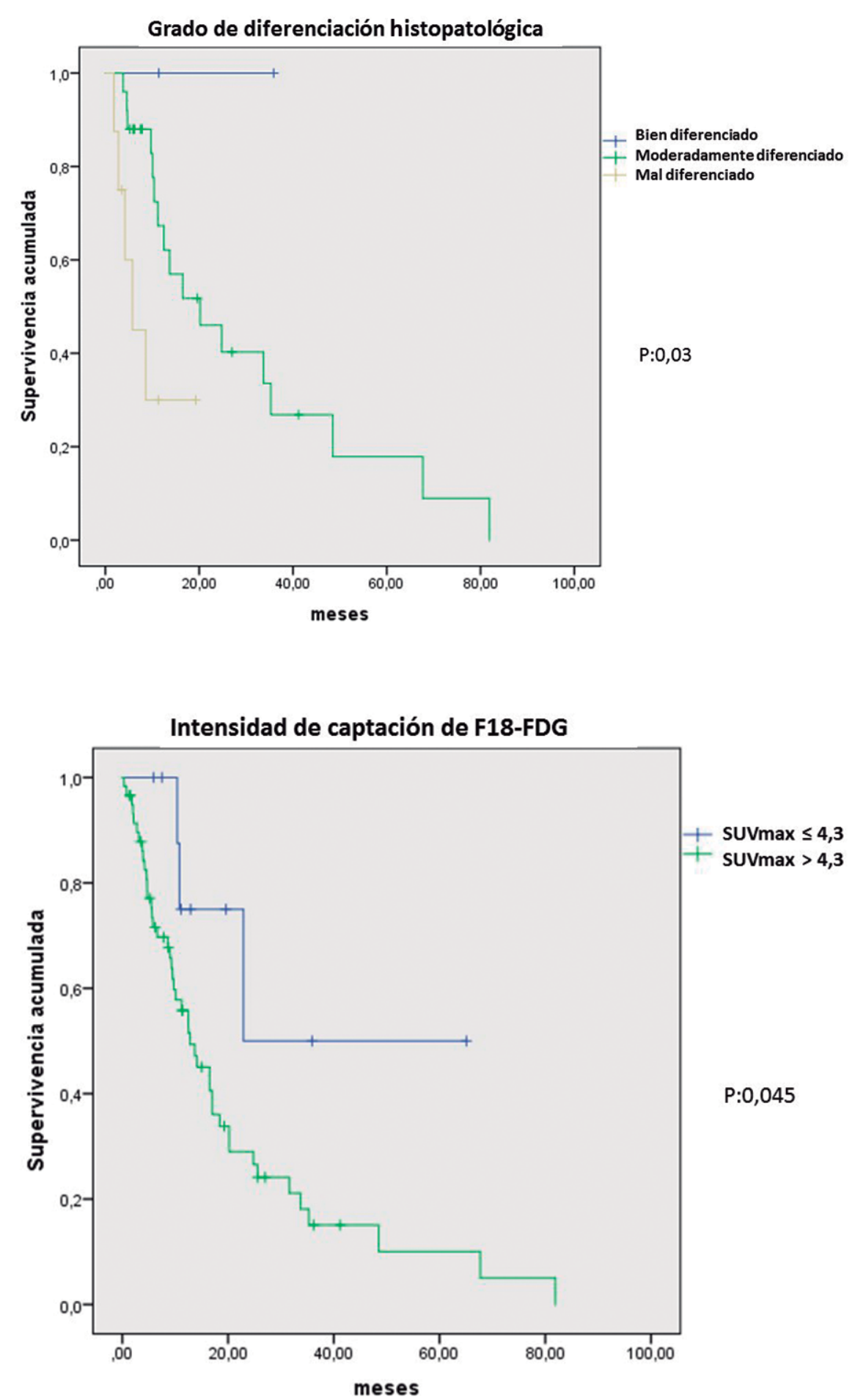

Figuras 4 y 5. Curvas de Kaplan-Meier según factor pronóstico. 
de FDG, pudieran requerir un tratamiento más agresivo, que además de la cirugía incorpore otras herramientas terapéuticas pre o postquirúrgicas, tanto terapias sistémicas como radioterapia del lecho quirúrgico ${ }^{19,20}$.

En 55 pacientes con cáncer de páncreas irresecable que se trataron con radioterapia estereotáxica, Schellenberg et $\mathrm{al}^{14}$ establecieron que aquellos tumores con SUVmax pretratamiento $\geq 6,2$ presentaban menor sobrevida que los de menor captación. En un estudio de 64 pacientes con cáncer de páncreas, Choi et $\mathrm{al}^{9}$ encontraron que un SUVmax > 3,5 y una pobre diferenciación tumoral conferían un peor pronóstico de sobrevida. Sperti et $\mathrm{al}^{10}$ calcularon el SUV de 60 pacientes con adenocarcinoma de páncreas, y determinaron que aquellos con $S U V \geq 4$ presentaron menor sobrevida. En el análisis multivariable ellos encontraron que el SUV y la etapa tumoral fueron los factores pronósticos más significativos. El valor de corte SUVmax de 4,3 obtenido en nuestra muestra es similar a los mencionados estudios, y la discreta variación en sus valores solo expresa las distintas condiciones en que fue obtenido (método de adquisición del PET/CT, variabilidad intrínseca de los distintos equipos y características propias de los pacientes).

La relativa similitud de valores del SUVmax respecto a lo publicado le confiere una robustez particular a nuestro estudio, y apoya la validez externa de sus resultados. Por otro lado, reafirma la necesidad de obtener resultados propios para cada centro, con valores de corte reales para una población y equipo PET/CT particulares, que legitimen su aplicación clínica.

Una de las fortalezas de nuestro estudio es la alta tasa de seguimiento de los pacientes, con acceso a la historia clínica antes y después del diagnóstico, a exámenes de laboratorio y test confirmatorios anatomopatológicos del tumor primario. Sin embargo, no se trata de un estudio pronóstico propiamente tal, ya que no es de naturaleza prospectiva ni se lleva a cabo un seguimiento sistemático. El punto de inicio corresponde al momento exacto del diagnóstico del cáncer, cuando es solicitado el PET/CT de etapificación, y el seguimiento se lleva a cabo de acuerdo a criterios clínicos, particulares para cada paciente, con punto de corte al momento de consulta del estado vital en el registro civil.

Una limitación de nuestro estudio es que la población incluida no representa necesariamente a la población general con cáncer de páncreas, ya que se incluyeron solo pacientes a los que se les realizó $\mathrm{PET} / \mathrm{CT}$, posiblemente con más factores de riesgo o con cuadros más complejos desde el punto de vista diagnóstico. Por otro lado, la condición socioeconómica y acceso a determinados sistemas de salud de la muestra puede diferir respecto a la población general de nuestro país, particularmente a aquella que se atiende en el sistema público. Estos sesgos de muestreo son factores que pudieran disminuir la validez externa de los resultados obtenidos. Sin embargo, la resecabilidad de $21,7 \%$ del grupo estudiado (15/69) y la frecuencia de metástasis ganglionares $(59,4 \%)$ y a distancia $(58 \%)$ encontradas, concuerdan perfectamente con lo descrito en la literatura ${ }^{4,19,20}$, lo que sugiere una adecuada representatividad de nuestra muestra respecto a los datos disponibles.

Como ya se mencionó anteriormente, otra limitación de nuestro estudio es el pequeño tamaño muestral, problema que se repite en otras publicaciones similares. Esto por supuesto disminuye la capacidad de encontrar significación estadística en la valoración de las variables estudiadas. Este hecho puede explicar en parte que algunos factores no resultaron significativos en nuestro estudio, pero sí mostraron utilidad pronóstica en otros, tales como el estadio $\mathrm{AJCC}^{10}$, tamaño tumoral ${ }^{13}$, la etapa $\mathrm{T}$ patológica ${ }^{13}$, el $\mathrm{VTM}^{12-14}$, el $\mathrm{MTG}^{12,13,21}$, y los niveles sanguíneos de Ca19-9 ${ }^{12}$.

Además de la utilidad pronóstica del PET/CT sugerida en nuestro estudio, es necesario, antes de recomendar su uso clínico en etapificación de cáncer de páncreas, determinar su valor diagnóstico, asunto que no ha sido demostrado suficientemente en la literatura, y que no formó parte de los objetivos de este trabajo.

\section{Conclusión}

El PET/CT proporciona información pronóstica de gran utilidad en pacientes con cáncer de páncreas, como la existencia de metástasis a distancia y la intensidad de captación de FDG por el tumor pancreático. Junto con el grado de diferenciación histológica, fueron los factores pronósticos más importantes en el grupo estudiado. 


\section{Referencias}

1. Siegel R, Ma J, Zou Z, Jemal A. Cancer statistics, 2014. CA Cancer J Clin 2014; 64: 9-29.

2. GLOBOCAN [Internet]. [citado el 1 de septiembre de 2017]. Recuperado a partir de: http://globocan.iarc.fr/

3. Conlon KC, Klimstra DS, Brennan MF. Long-term survival after curative resection for pancreatic ductal adenocarcinoma. Clinicopathologic analysis of 5-year survivors. Ann Surg 1996; 223: 273-7.

4. Michl P, Pauls S, Gress TM. Evidence-based diagnosis and staging of pancreatic cancer. Best Pract Res Clin Gastroenterol 2006; 20: 227-51.

5. Yamamoto T, Yagi S, Kinoshita H, Sakamoto Y, Okada $\mathrm{K}$, Uryuhara $\mathrm{K}$, et al. Long-term survival after resection of pancreatic cancer: A single-center retrospective analysis. World J Gastroenterol 2015; 21: 262-8.

6. Vincent A, Herman J, Schulick R, Hruban RH, Goggins M. Pancreatic cancer. Lancet Lond Engl 2011; 378: 60720.

7. National Comprehensive Cancer Network guidelines 2017. [citado el 1 de septiembre de 2017]. Recuperado a partir de: http://www.nccn.org/professionals/physician_gls/f_guidelines.asp\#site.

8. Moon SY, Joo KR, So YR, Lim JU, Cha JM, Shin HP, et al. Predictive value of maximum standardized uptake value (SUVmax) on 18F-FDG PET/CT in patients with locally advanced or metastatic pancreatic cancer. Clin Nucl Med 2013; 38: 778-83.

9. Choi HJ, Kang CM, Lee WJ, Song SY, Cho A, Mijin Yun M, et al. Prognostic Value of 18F-Fluorodeoxyglucose Positron Emission Tomography in Patients with Resectable Pancreatic Cancer. Yonsei Med J 2013; 54: 1377-83.

10. Sperti C, Pasquali C, Chierichetti F, Ferronato A, Decet G, Pedrazzoli S. 18-Fluorodeoxyglucose Positron Emission Tomography in Predicting Survival of Patients With Pancreatic Carcinoma. J Gastrointest Surg 2003; 7: 953-60.

11. Jadvar H, Alavi A, Gambhir SS. 18F-FDG Uptake in Lung, Breast, and Colon Cancers: Molecular Biology Correlates and Disease Characterization. J Nucl Med
2009; 50: 1820-7.

12. Xu H-X, Chen T, Wang W-Q, Wu C-T, Liu C, Long J, et al. Metabolic tumour burden assessed by 18F-FDG PET/ CT associated with serum CA19-9 predicts pancreatic cancer outcome after resection. Eur J Nucl Med Mol Imaging 2014; 41: 1093-102.

13. Lee JW, Kang CM, Choi HJ, Lee WJ, Song SY, Lee J-H, et al. Prognostic Value of Metabolic Tumor Volume and Total Lesion Glycolysis on Preoperative 18F-FDG PET/ CT in Patients with Pancreatic Cancer. J Nucl Med 2014; 55: 898-904.

14. Schellenberg D, Quon A, Minn AY, Graves EE, Kunz P, Ford JM, et al. 18Fluorodeoxyglucose PET is prognostic of progression-free and overall survival in locally advanced pancreas cancer treated with stereotactic radiotherapy. Int J Radiat Oncol Biol Phys 2010; 77: 1420-5.

15. Zhu D, Wang L, Zhang H, Chen J, Wang Y, Byanju S, et al. Prognostic value of 18F-FDG-PET/CT parameters in patients with pancreatic carcinoma. A systematic review and meta-analysis. Medicine 2017; 96: 33 (e7813): 1-11.

16. American Joint Comitte on Cancer. Pancreas Cancer Staging 7th edition [Internet]. [citado el 14 de Mayo de 2017]. Recuperado a partir de: https://cancerstaging.org/ references-tools/.../PancreasSmall.pdf.

17. Manterola C, Santander C, Otzen T. Cómo valorar e interpretar un artículo sobre pronóstico. Rev Chil Cir 2013; 65: 77-84.

18. Dooms C, van Baardwijk A, Verbeken E, MD, van Suylen RJ, Stroobants S, De Ruysscher D, et al. Association Between 18F-Fluoro-2-Deoxy-D-Glucose Uptake Values and Tumor Vitality: Prognostic Value of Positron Emission Tomography in Early-Stage Non-small Cell Lung Cancer. J Thorac Oncol 2009; 4: 822-8.

19. Vincent A, Herman J, Schulick R, Hruban RH, Goggins M. Pancreatic cancer. Lancet 2011; 378: 607-20.

20. Ansari D, Gustafsson A, Andersson R. Update on the management of pancreatic cancer: Surgery is not enough. World J Gastroenterol 2015; 21: 3157-65.

21. Chirindel A, Alluri KC, Chaudhry MA, Wahl RL, Pawlik TM, Herman JM, et al. Prognostic Value of FDG PET/ CT-Derived Parameters in Pancreatic Adenocarcinoma at Initial PET/CT Staging. AJR 2015; 204: 1093-9. 